\title{
Evaluation of ultrasonography in fetal intestinal malrotation with midgut volvulus
}

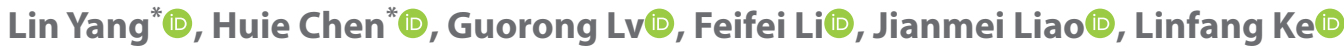 \\ Zhangzhou Affiliated Hospital of Fujian Medical University, Zhangzhou, China \\ ${ }^{*}$ these authors contribute equally to this work
}

\begin{abstract}
Objectives: To investigate the clinical significance of prenatal diagnosis and prognostic evaluation of fetal intestinal malrotation with midgut volvulus via ultrasonography.

Material and methods: Ultrasonographic findings and clinical outcomes of fetal intestinal malrotation with midgut volvulus prenatally diagnosed via ultrasound at the Zhangzhou Hospital Affiliated of Fujian Medical University from January 2013 to May 2020 were summarised and analyzed.

Results: Eleven cases of fetal intestinal malrotation with midgut volvulus were accurately prenatally diagnosed according to the specific ultrasound signs, such as 'whirlpool sign' and 'twining sign'. Indirect and nonspecific ultrasonographic signs included ascites in four cases and echogenic bowel in nine, all of which were accompanied by intestinal dilatation at various degrees. Among all cases, two were complicated with other system abnormalities, and one had a chromosome abnormality. Three pregnant women chose termination of pregnancy, and eight neonates were transferred to the pediatric department for surgery after obtaining written informed consent. An accurate prenatal diagnosis was confirmed postoperatively, and the fetus recovered well postoperatively.

Conclusions: Fetal intestinal malrotation with midgut volvulus has typical sonographic features, and ultrasonography is the method of choice for prenatal diagnosis of this disease. Fetal intestinal malrotation with midgut volvulus is a non-fatal congenital malformation that can be treated. Prenatal diagnosis is essential for early postnatal treatment, and early surgery can often obtain good efficacy and prognosis.
\end{abstract}

Key words: ultrasonography; prenatal; whirlpool sign; intestinal malrotation; midgut volvulus

Ginekologia Polska 2022; 93, 4: 296-301

\section{INTRODUCTION}

Congenital intestinal malrotation is a congenital intestinal malformation caused by abnormal rotation of the superior mesenteric artery (SMA) on the axis during embryonic intestinal development, with an incidence of 1 in 10.000 newborns [1]. Variation in bowel position and mesenteric attachment results in the duodenum's compression by the umbilical cord, leading to obstruction and midgut volvulus. Intestinal malrotation can lead to midgut volvulus in children at any stage before or after birth, resulting in complete or incomplete intestinal obstruction. Clinical manifestations are recurrent intermittent vomiting or frequent vomiting, abdominal distension, fullness, bloody stools, fever, intestinal perforation when complicated by intestinal blood flow disorders, and metabolic acidosis. Its lack of specificity is easily misdiagnosed and mistreated and can even be life-threatening [2]. Neonatal intestinal malrotation with midgut volvulus is frequently reported, but it is rare in the fetus. Progress in prenatal ultrasound diagnosis has led to occasional detection of fetal intestinal malrotation with midgut volvulus [3].

Ultrasonography has unique advantages in diagnosing fetal gastrointestinal malformations. The degree of intestinal dilatation and the SMA and superior mesenteric vein (SMV) location can be dynamically observed on two-dimensional (2D) images by color Doppler technique. The'whirlpool sign' is the main sign of ultrasound diagnosis of the disease: the formation of whirlpool mass with SMA as axis, spiral torsion of intestinal lumen, and mesentery accompanied by vessels is the pathological basis of ultrasonographic findings of intestinal malrotation with midgut volvulus [2]. In this study, we reported 11 cases of fetal intestinal malrotation

\section{Corresponding author:}

Lin Yang

Zhangzhou Affiliated Hospital of Fujian Medical University, Building 59\#, Shengli West Road, Zhangzhou, 363000 Zhangzhou, China

e-mail: linyang4043@163.com 
with midgut volvulus diagnosed prenatally in our hospital and their postnatal follow-up results. The ultrasonographic features, prenatal diagnosis, and prognosis analysis were discussed in combination with a literature review to provide evidence for clinical intervention.

\section{MATERIAL AND METHODS Participants}

From January 2013 to May 2020, eleven cases of fetal intestinal malrotation with midgut volvulus were detected in 86.327 pregnant women via prenatal ultrasonography at the Zhangzhou Hospital Affiliated of Fujian Medical University, with an incidence of $0.013 \%(11 / 86.327)$. The pregnant women's mean age was $28.2 \pm 3.6$ (range, $22-35$ ) years. The gestational age at the first detection of midgut volvulus by prenatal ultrasonography was $30.7 \pm 3.1$ (range, 25-35) weeks.

\section{Instruments and methods}

The GE Voluson E8 or GE-730pro color Doppler ultrasound diagnostic instrument (General Electric Co., USA) was used, with the convex array probe of $3.5 \sim 6.0 \mathrm{MHz}$. First, the fetus undergoes systematic obstetric ultrasound screening followed by a multi-section scan of the fetal abdomen. The 'whirlpool sign' of intestinal malrotation was frequently observed in the middle and upper gastrointestinal tract. The positional relationship and walking direction of SMA and SMV and the 'whirlpool sign' or 'twinning sign' of blood vessels were observed using color Doppler flow imaging, suggesting the intestinal diagnosis malrotation with midgut volvulus. Abnormal abdominal ultrasonographic signs, such as bowel dilatation, ascites, intestinal bowel echogenicity, and pseudocysts, were also detected.

All fetuses diagnosed with intestinal malrotation with midgut volvulus by ultrasound were followed up until postpartum. Fetal postnatal diagnosis, postnatal surgery, and postoperative health status were also followed up. Monitoring of the fetuses were conducted every two weeks.

\section{RESULTS \\ Results of prenatal ultrasonography in 11 fetuses}

Prenatal ultrasonography showed a moderately echogenic mass in the upper abdomen with a 'whirlpool sign' in all 11 cases of fetal intestinal malrotation with midgut volvulus (Fig. 1A). Colour Doppler flow imaging revealed a 'twinning sign' of blood vessels formed by the SMV surrounding the SMA's root in the mass (Fig. 1B). The coronal section of the mass was obtained by rotating the probe horizontally at the root of the SMA of the descending aorta and revealed that the SMV twined around the SMA, presenting a 'whirlpool sign' of the SMV and SMA (Fig. 1C) with abdominal intestinal dilatation at various degrees (Fig. 1D). The degree of expansion varied according to disease development. Ultrasonography revealed increased intestinal echogenicity in nine cases (Fig. 1E) and ascites in four patients (Fig. 1F). Other organs were systematically examined by ultrasound in 11 cases. One fetus was complicated by a ventricular septal defect (VSD) and hexadactyly, while the other was complicated by renal duplication. No other systemic abnormalities were found in any of the other cases.
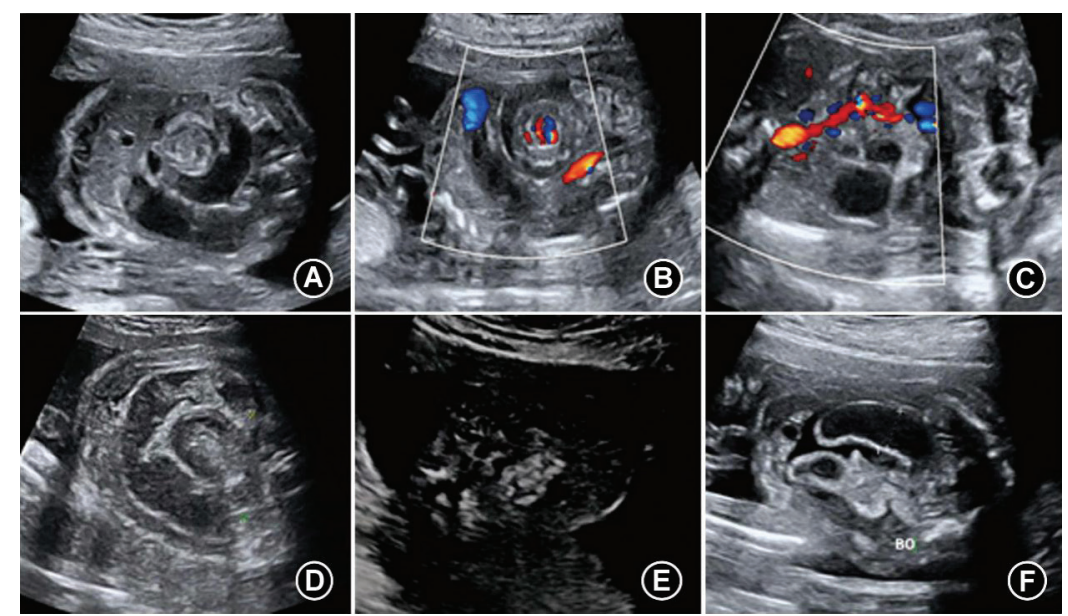

Figure 1. Prenatal ultrasonograms of fetal intestinal malrotation with midgut volvulus; A — dilated and twisted bowel loops showed a spiral twist around the superior mesenteric artery, presenting a typical 'whirlpool sign'; B — the blood flow 'whirlpool sign' formed by the SMV surrounding the SMA's spiral in the mass; C — 'twinning sign' of SMA and SMV revealed by color Doppler flow imaging; $\mathbf{D}$ - intestinal obstruction due to fetal intestinal malrotation with midgut volvulus leads to marked dilatation of the intestine with a small number of ascites; $\mathbf{E}-$ fetuses with intestinal malrotation and midgut volvulus showed increased echogenicity of the abdominal bowel; F — fetal intestinal malrotation with midgut volvulus showed apparent dilation of the intestine with many ascites 


\section{Clinical outcomes and follow-up results in 11 fetuses}

Eleven gastrointestinal malrotation cases with midgut volvulus were prenatally screened using ultrasonography and confirmed after termination of pregnancy or delivery. Among these cases, one case of fetal karyotype showed trisomy 21 syndromes in amniocentesis, whereas no abnormalities were found in the karyotypes of the other 10 cases. According to the Chinese law, termination of pregnancy may be performed if (a) the fetus is suffering from a serious genetic disease; (b) the fetus has serious defects; (c) due to serious diseases, continued pregnancy may endanger the life of the pregnant woman or seriously endanger the health of pregnant women. After giving written informed consent, three pregnant women chose termination of pregnancy. The fetuses were confirmed by autopsy to be associated with varying intestinal dilatation, increased intestinal echogenicity, and ascites. Because of an induction of labor for increased bowel dilatation, three pregant women underwent preterm delivery by cesarean section at about 34 weeks of gestation after signing informed consent. All the three neonates presented with abdominal cyanosis, edema, and respiratory instability and were transferred to pediatrics for exploratory laparotomy. Five pregnant women underwear full-term delivery at about 38 weeks of gestation. All the five neonates were transferred to pediatric for surgery after birth and were associated with varying degrees of bowel dilatation and increased bowel echogenicity. All the eight neonates recovered well after surgery.

Fetal intestinal malrotation with midgut volvulus was found intraoperatively, accompanied by segmental intestinal atresia at various degrees. Three fetuses developed temporary short bowel syndrome after surgery and were followed up for two years after birth. They showed no significant difference in digestive function compared with normal infants and no significant developmental abnormalities. Table 1 presented the pre-operative ultrasound findings, obstetric treatment, and postoperative follow-up results of 11 cases of fetal intestinal malrotation with midgut volvulus.

\section{DISCUSSION}

In 1898, Mall first described the embryological manifestations of intestinal malrotation [4]. During the embryonic period, regular rotation and midgut fixation occur mainly at 4-12 weeks of gestation. At 5-6 weeks of the embryo's age, the midgut, the abdominal cavity is relatively small, and loops protrude into the umbilical body cavity to form a physiological midgut hernia. The midgut continues to grow in the umbilical coelom and rotates $90^{\circ}$ anticlockwise with the SMA as the axis so that the intestinal loop changes from sagittal position to horizontal position. After ten weeks, the abdominal cavity grows rapidly, and the midgut gradually retracts into the abdominal cavity and continuously rotates $180^{\circ}$ anticlockwise. At that time, the SMV is located on the right side of the SMA. The duodenum bypasses the posterior SMA to the left, and the duodenal ligamnt attaches to the posterior abdominal wall from the upper left to the lower right corner. Subsequently, the jejunum and ileum reach the middle of the abdominal cavity. The cecum rotates the right lower abdomen. The ascending and descending colon attaches to the posterior abdominal wall from the mesocolon [5]. During this process, the interruption may lead to the intestine's malrotation, resulting in an abnormal anatomical position of the intestine, leading to various pathological conditions. Intestinal malrotation and incomplete mesenteric attachment lead to the small intestinal mesentery becoming suspended in the retroperitoneum by the narrow SMA root. The small intestine's high level of motility predisposes it to a volvulus with an SMA axis, leading to interruption of blood flow and ischemic injury [6].

In this study, fetal intestinal malrotation with midgut volvulus was detected at a gestational age of $25+5$ to $34+5$. Most abnormalities were found via ultrasound in late pregnancy, which coincided with fetal intestinal development. It is difficult to distinguish the small intestine, colon, and rectum earlier than 20 weeks of gestation due to lack of fetal intestinal contents, collapsed bowel, and unclear wall structure. At around 24 weeks of gestation, ultrasonography can accurately distinguish the small fetal intestine and colon echoes.

Due to meconium's presence, the rectum and colon are usually hypoechoic after 24 weeks of gestation, and the left descending colon is generally identifiable. The transverse and right ascending colon can be identified before 31 weeks of gestation in about $50 \%$ of fetuses. After 33 weeks of gestation, the jejunum is hypoechoic due to the influx of amniotic fluid [7]. These specific ultrasonographic findings are valuable for the accurate diagnosis of intestinal malformations. Intestinal malrotation with midgut volvulus has significant abnormal ultrasonographic findings, which can be clearly and accurately observed using 2D ultrasound and color Doppler flow imaging [8]. The diagnosis was confirmed mainly by showing the walking of the intestine, mesentery, and mesenteric vessels and the involvement of the intestine. The 'whirlpool sign' refers to a whirlpool-like mass formed by the spiral volvulus of the intestinal cavity with the SMA as the axis, accompanied by the mesenteric vessels. On the other hand, the 'twining sign' is caused by an abnormal spatial relationship between the SMV and SMA and clockwise twining of the SMV and dilated intestine around the SMA $[5,6,9]$. The 'whirlpool sign' and 'twining sign' have recently been recognized as direct signs of fetal intestinal malrotation with midgut volvulus [10]. In this study, 11 fetuses were diagnosed by 'whirlpool sign' and 'twinning sign'. 


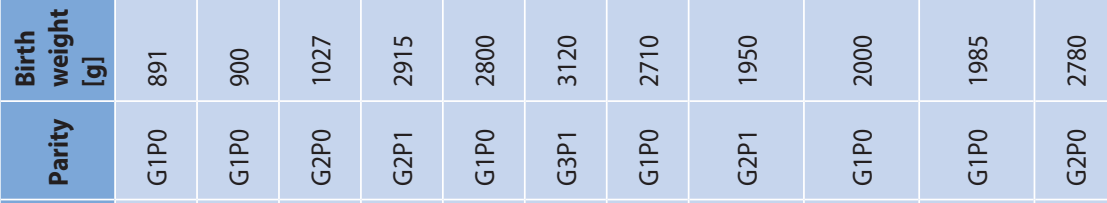

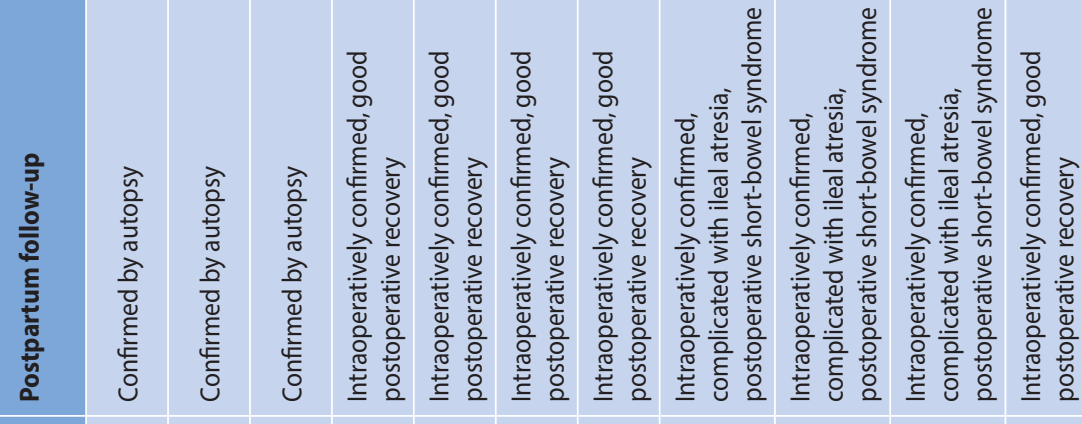

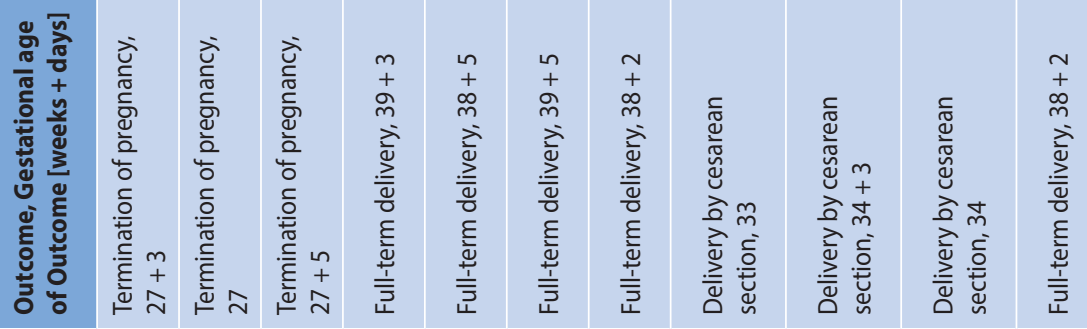

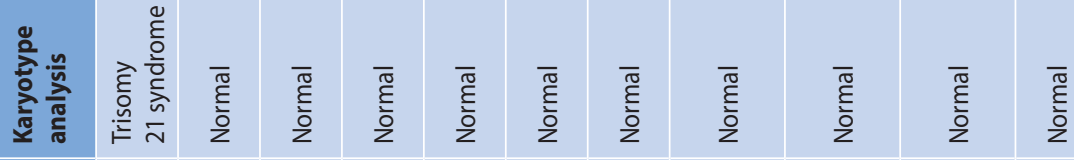

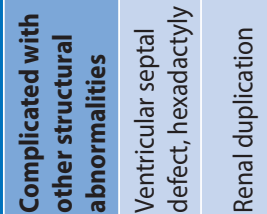

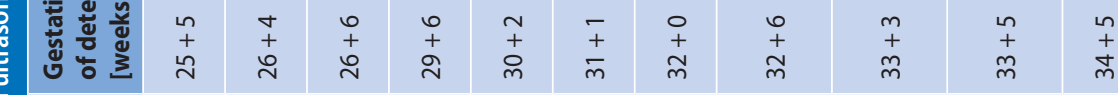

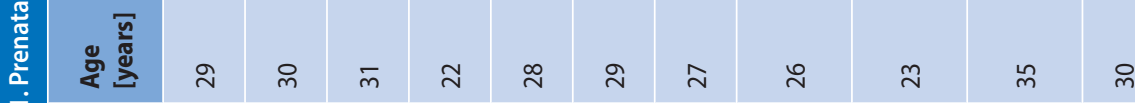

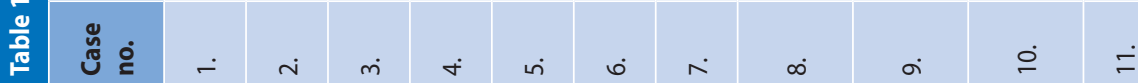


Indirect and nonspecific ultrasonographic signs of intestinal malrotation with midgut volvulus include intestinal dilatation, increased intestinal echogenicity, ascites, pseudocysts, and hyperhydramnios. These signs are mainly due to reduced fetal swallowing caused by volvulus or viscous meconium in the intestine caused by slowed fetal intestinal peristalsis. Intestinal ischemia and necrosis may occur when volvulus leads to mesenteric vascular obstruction. This may lead to fetal fecal peritonitis, intestinal obstruction, and even intestinal perforation with various echogenic features at different disease stages [11]. In the present study, all $11 \mathrm{fe-}$ tuses were associated with varying degrees of intestinal dilatation, nine showed increased intestinal echogenicity on ultrasonography, and four showed varying degrees of ascites on ultrasonography. This further demonstrates that the combination of direct and indirect signs indicates intestinal malrotation with midgut volvulus.

Ladd's surgery for intestinal malrotation is recommended, and early surgery is strongly recommended due to its positive effects. This treatment aims to restore the distorted mesentery and remove the adhesion cord to reconstruct the digestive tract [12]. Eleven fetuses with intestinal malrotation and midgut volvulus were detected in this study. Except for three pregnant women who required termination of pregnancy, eight neonates underwent Ladd's surgery. Three of the newborns were found to have concomitant small intestinal atresia during surgery and were further treated with intestinal resection and anastomosis. After surgery, these three neonates developed short bowel syndrome did not require parenteral nutrition after six months of nutritional support, and were discharged. It has been reported that midgut volvulus can cause local blood circulation disorders in the intestine during fetal life, leading to aseptic necrosis of the intestine, which is an essential factor causing jejunoileal atresia. Therefore, intestinal atresia should be one of the significant intestinal malrotation complications with midgut volvulus [11]. The results of the two-year postoperative follow-up of eight fetuses showed no difference in digestive function compared with normal infants. Moreover, blood routine or liver and kidney function revealed no abnormalities, and there were no apparent abnormalities in growth and development.

Congenital intestinal malrotation with midgut volvulus can exist as a solitary malformation, but it is mostly associated with other malformations. Congenital intestinal malrotation has been highlighted as a component of multiple systemic malformations [13]. The incidence of intestinal malrotation combined with other malformations, such as the central nervous system, heart, and genitourinary system, has been reported to be 25-30\% [14]. Of the 11 fetuses in this study, two (18\%) had other systemic malformations. One fetus had VSD and hexadactyly with karyotype showing trisomy 21, while the other fetus had renal duplication with normal karyotype. No abnormal karyotype was found in the other nine cases of intestinal malrotation with midgut volvulus. These results suggested that the fetus should be screened for systemic structural malformations when intestinal malformations are detected by ultrasound. When other systemic structural malformations of the fetus are detected by ultrasound, the intestine should be carefully examined to avoid misdiagnosis. Additionally, when fetal intestinal malrotation with midgut volvulus is found, amniocentesis and karyotype analysis should be routinely recommended for pregnant women. These examinations are highly valuable to evaluate fetal prognosis.

Congenital intestinal malrotation with midgut volvulus can lead to fetal intestinal necrosis, atresia, and even complicated fetal fecal peritonitis during intestinal perforation seriously threatens perinatal life. Although volvulus and necrosis cause massive fluid leakage from the abdominal cavity, electrolyte disturbances do not occur in the fetus through placental compensation because the fetal intestine is in a sterile environment. However, the situation after birth will be significantly different. Therefore, when intestinal malrotation with midgut volvulus is confirmed prenatally, accompanied by massive ascites, the pregnancy should be terminated as soon as possible. The fetus should be transferred to pediatrics for surgery. Prompt surgery should be performed when the fetus has no evidence of severe infection, electrolyte disturbances, or malnutrition [15]. Recent developments in surgical techniques and improvements in corresponding treatment options have significantly improved the survival rate of fetal intestinal malrotation with midgut volvulus.

Our follow-up data indicated a good fetal prognosis after surgical treatment. Therefore, we believe that termination of pregnancy should be avoided after prenatal diagnosis and consultation with a high suspicion of fetal congenital intestinal malrotation with midgut volvulus, and pregnant women should be instructed to follow-up closely to rule out other fetal abnormalities. The fetus should be treated as soon as possible after birth under the guidance of a pediatrician.

\section{CONCLUSIONS}

In conclusion, fetal intestinal malrotation with midgut volvulus has characteristic ultrasonographic signs, and ultrasonography should be the method of choice for prenatal diagnosis of this disease. Ultrasonographic diagnosis of fetal intestinal malrotation with midgut volvulus plays an essential role in guiding obstetric management and early neonatal treatment. In this study, due to the small sample size, a detailed ultrasound analysis of congenital intestinal malrotation with midgut volvulus complicated by intestinal atresia could not be performed. Moreover, the results of 
other systemic malformations and chromosomal screening were slightly different from those of previous studies due to the small sample size. Therefore, future studies including more cases are needed.

\section{Conflict of interest}

All authors declare no conflict of interest.

\section{REFERENCES}

1. Lin JN, Lou CC, Wang KL. Intestinal malrotation and midgut volvulus: a 15-year review. J Formos Med Assoc. 1995; 94(4): 178-181, indexed in Pubmed: 7606179.

2. Liu S, Wu Q. Ultrasonographic diagnosis of intestinal malrotation with midgut volvulus in fetus. Chinese Journal of Medical Ultrasound (Electronic Edition). 2016; 13(11): 840-844, doi: 10.1148/cases.20214270.

3. Yilmaz Y, Demirel G, Ulu HO, et al. Urgent surgical management of a prenatally diagnosed midgut volvulus with malrotation. Eur Rev Med Pharmacol Sci. 2012; 16 Suppl 4: 52-54, indexed in Pubmed: 23090808.

4. Sciarrone A, Teruzzi E, Pertusio A, et al. Fetal midgut volvulus: report of eight cases. J Matern Fetal Neonatal Med. 2016; 29(8): 1322-1327, doi: 10.3109/14767058.2015.1047336, indexed in Pubmed: 26103780

5. Jakhere SG, Saifi SA, Ranwaka AA. Fetal small bowel volvulus without malrotation: the whirlpool \& coffee bean signs. J Neonatal Perinatal Med. 2014; 7(2): 143-146, doi: 10.3233/NPM-1476013, indexed in Pubmed: 25104121.

6. KargI S, Wagner O, Pumberger W. Volvulus without malposition - a single-center experience. J Surg Res. 2015; 193(1): 295-299, doi: 10.1016/j. jss.2014.08.043, indexed in Pubmed: 25266606.
7. Kornacki J, Czarnecka M, Błaszczyński M, et al. Congenital midgut volvulus associated with fetal anemia. Fetal Diagn Ther. 2010; 28(2): 119-122, doi: 10.1159/000315051, indexed in Pubmed: 20616522.

8. Has R, Gunay S. 'Whirlpool'sign in the prenatal diagnosis of intestinal volvulus. Ultrasound Obstet Gynecol. 2002; 20(3): 307-308, doi: 10.1046/j.1 469-0705.2002.00779 4.x, indexed in Pubmed: 12230462.

9. Pracros JP, Sann L, Genin G, et al. Ultrasound diagnosis of midgut volvulus: the "whirlpool" sign. Pediatr Radiol. 1992; 22(1): 18-20, doi: 10.1007/BF02011603, indexed in Pubmed: 1594304.

10. Tang $X$, Tao R, Zhang X, et al. Assessment of fetal superior mesenteric artery and vein by three-dimensional power Doppler sonography. Zhejiang Da Xue Xue Bao Yi Xue Ban. 2019; 48(4): 453-458, indexed in Pubmed: 31901052

11. Noreldeen SA, Hodgett SG, Venkat-Raman N. Midgut volvulus with hemorrhagic ascites: a rare cause of fetal anemia. Ultrasound Obstet Gynecol. 2008; 31(3): 352-354, doi: 10.1002/uog.5223, indexed in Pubmed: 18167627

12. Ooms N, Matthyssens LEM, Draaisma JM, et al. Laparoscopic treatment of intestinal malrotation in children. Eur J Pediatr Surg. 2016; 26(4): 376-381, doi: 10.1055/s-0035-1554914, indexed in Pubmed: 26086418

13. Sulkowski JP, Cooper JN, Duggan EM, et al. Early versus delayed surgical correction of malrotation in children with critical congenital heart disease. J Pediatr Surg. 2015; 50(1): 86-91, doi: 10.1016/j.jpedsurg.2014.10.006, indexed in Pubmed: 25598100.

14. Ezer SS, Oguzkurt $P$, Temiz A, et al. Intestinal malrotation needs immediate consideration and investigation. Pediatr Int. 2016; 58(11): 1200-1204, doi: 10.1111/ped.13075, indexed in Pubmed: 27353636.

15. Vassaur J, Vassaur H, Buckley FP. Single-incision laparoscopic Ladd's procedure for intestinal malrotation. JSLS. 2014; 18(1): 132-135, doi: 10.4293/108680813X13654754534954, indexed in Pubmed: 24680157. 